\title{
Learning-Dependent Increases in Sleep Spindle Density
}

\author{
Steffen Gais, Matthias Mölle, Kay Helms, and Jan Born \\ Department of Neuroendocrinology, University of Lübeck, 23564 Lübeck, Germany
}

\begin{abstract}
Declarative memory consolidation is enhanced by sleep. In the investigation of underlying mechanisms, mainly rapid eye movement (REM) sleep and slow-wave sleep have been considered. More recently, sleep stage 2 with sleep spindles as a most prominent feature has received increasing attention. Specifically, in rats hippocampal ripples were found to occur in temporal proximity to cortical sleep spindles, indicating an information transfer between the hippocampus and neocortex, which is supposed to underlie the consolidation of declarative memories during sleep. This study in humans looks at the changes in EEG activity during nocturnal sleep after extensive training on a declarative learning task, as compared with a nonlearning control task of equal visual stimulation and subjectively rated cognitive strain. Time spent in each sleep stage,
\end{abstract}

A series of recent studies described a multifaceted relationship between sleep and memory. Recall performance on some memory tasks, mainly those tasks depending on the hippocampusdependent declarative memory system, is enhanced after "early" sleep from the first half of the night in which slow-wave sleep is predominant (Plihal and Born, 1997). Nondeclarative types of memory can also benefit from early sleep, although in general they profit to a greater extent from the second, rapid eye movement (REM) sleep-rich half of the night (Plihal and Born, 1999; Gais et al., 2000). Although the beneficial effect of sleep on memory performance seems to be indubitable, the underlying mechanisms mediating consolidation and reorganization of memories during sleep are still unclear. Apart from neuromodulator activity patterns during different sleep stages, electrophysiological features of sleep are being discussed as possible origins for sleep-related memory improvement. Initially, mainly EEG activity during REM sleep has been suspected to reflect memory reprocessing because of its wake-like pattern and the occurrence of rhythmic theta activity. However, in the last decade non-REM sleep, including slow-wave sleep (SWS) and sleep stage 2 (S2), moved to the center of attention (Steriade et al., 1993a; Smith and MacNeill, 1994; Giuditta et al., 1995; Meier-Koll et al., 1999).

Regarding declarative memory consolidation, sleep may provide a state during which newly acquired memory contents, which are temporarily stored in the hippocampus, can be easily transferred to the neocortex for integration into long-term memories (Buzsaki, 1998; Hasselmo, 1999; Sutherland and McNaughton, 2000). According to this concept, during the wake state new

\footnotetext{
Received March 27, 2002; revised May 21, 2002; accepted May 24, 2002.

This work was supported by a grant of the Deutsche Forschungsgemeinschaft.

Correspondence should be addressed to Dr. Jan Born, Department of Neuroendocrinology, University of Lübeck, Ratzeburger Allee 160, 23564 Lübeck, Germany. E-mail: born@kfg.mu-luebeck.de.

Copyright (C) 2002 Society for Neuroscience $\quad 0270-6474 / 02 / 226830-05 \$ 15.00 / 0$
}

spindle density, and EEG power spectra for 28 electrode locations were determined. During sleep after training, the density of sleep spindles was significantly higher after the learning task as compared with the nonlearning control task. This effect was largest during the first 90 min of sleep ( $p<0.01$ ). Additionally, spindle density was correlated to recall performance both before and after sleep $(r=0.56 ; p<0.05)$. Power spectra and time spent in sleep stages did not differ between learning and nonlearning conditions. Results indicate that spindle activity during non-REM sleep is sensitive to previous learning experience.

Key words: sleep spindles; learning; declarative memory; sleep; EEG; human

information enters the hippocampal CA3 region through the entorhinal cortex, where it can be stored temporarily without disturbing previously acquired memories. During non-REM sleep the flow of information is reversed, and hippocampal efferents to the neocortex become predominant. Studies in rats have demonstrated the existence of hippocampal cells showing a replay of previous activation patterns during non-REM sleep (Kudrimoti et al., 1999; Nadasdy et al., 1999) and a coordination of hippocampal and neocortical reactivation (Qin et al., 1997). In particular, sharp wave-ripple pattern activity, i.e., massive excitatory activity from CA3 with superimposed $\sim 200$ $\mathrm{Hz}$ oscillations, is transferred via the entorhinal cortex to the neocortex (Ylinen et al., 1995; Qin et al., 1997; Sutherland and McNaughton, 2000). Therefore, sharp wave-ripple patterns are a good candidate for being the basis of hippocampalneocortical memory transfer and underlying the integration and long-term storage of newly acquired material (Chrobak and Buzsaki, 1996). A neocortical correlate of hippocampal ripple activity was found by Siapas and Wilson (1998), who showed that ripples occur in a temporal correlation to sleep spindles in single cell derivations. Here, we provide evidence in humans for an increased sleep spindle activity after extensive training of an intentional declarative learning task, as compared with a nonlearning task of equal visual stimulation and cognitive strain. The data thus provide further indication of an association between the hippocampal memory system and the mechanisms generating sleep spindles.

\section{MATERIALS AND METHODS}

Subjects and procedures. A total of 16 subjects ( 8 male, 8 female) participated in this study. They were nonsmokers, aged 18-30 years (mean, 24.8 years), and regular sleepers with no disturbance of their sleep-wake rhythm during the 6 weeks before the experiment. Subjects stayed for three nights in the sleep laboratory, sleeping from 11:00 P.M. to 7:00 
A.M. The first night served only to adapt the subject to sleeping in the laboratory with EEG electrodes attached. Nights two and three were separated by at least $7 \mathrm{~d}$. The experiments were approved by the ethics committee of the University of Lübeck.

Tasks. During the second and third nights, directly before going to sleep (i.e., between 9:30 and 10:30 P.M.) subjects performed in balanced order one of two cognitive tasks. In the learning task, subjects had to learn paired-associate lists of 336 unrelated words, arranged in 21 groups of 8 pairs. Each group of words was presented twice for 106 and $70 \mathrm{sec}$, respectively, resulting in a total learning time of $60 \mathrm{~min}$. The second presentation of the list had a shorter duration to hold the difficulty for the subjects at a constant level throughout the experiment. Both presentations of the list were separated by a break of $2 \mathrm{~min}$. To make the mnemonic strategies more comparable, subjects were instructed to visually imagine a relation of the two otherwise unrelated words of each pair. Words were selected for high concreteness, low emotionality, and word length (5-11 letters). The length of the word list was chosen in prestudies, so that subjects were able to retain $60-90 \%$ of the words. Recall performance was tested directly after learning as well as on the next morning, $\sim 30$ min after awakening. Recall was cued, i.e., subjects were presented with the first word of each pair and had to recall the second one. The nonlearning task was designed to resemble the learning task in as many ways as possible but without the intentional learning component. We chose a task where subjects were instructed to count all letters containing curved lines (e.g., J, P, U, but not W, Y, K) on word-pair stimulus displays identical to those used for the learning task. Therefore, visual input and task duration were equal in both conditions, but subjects had no possibility to semantically process the words. Another criterion for the selection of the tasks was their equal cognitive demand. Subjective cognitive strain, task difficulty, wearisomeness, and boringness of both tasks were rated on five-point scales by the subjects in pre-studies and in the experiment proper directly after completing the task. Additionally, subjects rated tiredness, sleepiness, and tenseness after completing the task.

EEG recordings and analysis. During sleep, EEG was recorded digitally on a Synamps EEG amplifier (NeuroScan Inc. Sterling, VA) using $\mathrm{Ag}-\mathrm{AgCl}$ electrodes with $500 \mathrm{~Hz}$ sampling frequency, a $0.15 \mathrm{~Hz}$ highpass filter, a $70 \mathrm{~Hz}$ low-pass filter, and a $50 \mathrm{~Hz}$ notch filter. Electrodes were placed according to an extended 10-20-System with 28 channels and referenced to linked mastoids. Additionally, horizontal and vertical eye movements and the electromyogram (chin and neck) were recorded. Each $30 \mathrm{sec}$ epoch of sleep was scored visually according to standard criteria (Rechtschaffen and Kales, 1968). Data from three subjects were excluded from further analyses, because of poor sleep quality or bad quality of the EEG record. For sleep spindle detection, ocular artifacts were removed automatically (Gratton et al., 1983), and all epochs containing movement artifacts were excluded from analysis. Spindles were counted by means of an automatic algorithm that used the following steps: (1) filtering the EEG with a $12-15 \mathrm{~Hz}$ bandpass filter, (2) calculating the root mean square (RMS) of each $100 \mathrm{msec}$ interval of the filtered signal, (3) counting the number of times the RMS power crossed a constant detection threshold of $10 \mu \mathrm{V}$ for $0.5-3 \mathrm{sec}$. This algorithm correctly identified $>95 \%$ of the spindles detected by visual scoring of experienced raters. Also, individual differences in spindle power are negligible because all statistical tests were within-subject comparisons. (4) Spindle density was calculated as the mean number of spindles per 30 sec epoch. Spindle detection was performed in Matlab. Because spindle activity in the human EEG can be measured best on midline electrode positions, this report focuses on results from frontocentral $(\mathrm{Fz})$ and central $(\mathrm{Cz})$ electrodes. Analysis of other electrode positions did not add essentially to the interpretation of data. Statistics relied on a doublesided paired sample $t$ test, with results of $p \leq 0.05$ considered as significant.

Additionally, power spectral analyses were performed for all recorded channels. For these analyses, the sample rate was transformed to $100 \mathrm{~Hz}$ after digitally filtering the EEG signal with a $30 \mathrm{~Hz}$ low pass filter, and a Fast Fourier Transform was performed for every $5.12 \mathrm{sec}$ interval. Results were averaged over all epochs of S2, SWS (i.e., sleep stage 3 and 4) and REM sleep, respectively, which did not contain movement artifacts, and standard frequency bands were computed $(\delta: 1-4 \mathrm{~Hz} ; \theta: 4-8$ $\mathrm{Hz} ; \alpha$ : 8-12 Hz; $\sigma: 12-15 \mathrm{~Hz} ; \beta$ : $15-20 \mathrm{~Hz}$ ). Statistical analyses relied on ANOVA with three within-subject factors (learning vs nonlearning, sleep stage, and frequency band). Additionally, $t$ tests for paired samples were performed for all frequency bands, channels, and sleep stages.
Table 1. Percentage of time spent in each sleep stage (mean \pm SEM) after learning and nonlearning conditions.

\begin{tabular}{lrr} 
Sleep stage & \multicolumn{1}{c}{ Learning } & Nonlearning* \\
\hline W & $3.0 \pm 1.2 \%$ & $1.1 \pm 0.4 \%$ \\
S1 & $4.2 \pm 0.9 \%$ & $4.7 \pm 0.8 \%$ \\
S2 & $56.3 \pm 1.7 \%$ & $56.0 \pm 1.1 \%$ \\
S3 & $9.4 \pm 1.3 \%$ & $10.2 \pm 1.2 \%$ \\
S4 & $7.3 \pm 1.1 \%$ & $7.3 \pm 1.1 \%$ \\
REM & $19.0 \pm 1.7 \%$ & $19.9 \pm 1.4 \%$ \\
SOL & $13.6 \pm 3.3 \mathrm{~min}$ & $12.3 \pm 2.8 \mathrm{~min}$ \\
\hline
\end{tabular}

*All pairwise comparisons are nonsignificant $(p>0.30)$. W, Wake; S1-S4, nonREM sleep stages 1-4; REM, rapid eye movement sleep; SOL, sleep onset latency.

\section{RESULTS}

The learning rate was found to be in the expected margin with a mean $74 \pm 5 \%$ (mean \pm SEM) of correctly recalled words directly after learning as well as in the next morning. Sleep parameters were identical in both conditions (Table 1). Both the learning and the nonlearning task were rated equally regarding difficulty ( $3.0 \pm 0.4$ vs $2.9 \pm 0.3$ on 5-point scales), cognitive strain (both conditions $4.0 \pm 0.2$ ), and wearisomeness $(3.8 \pm 0.3$ vs $3.9 \pm 0.2$; all ratings nonsignificant, $p>0.75)$. As expected, reflecting probably the more semantic nature of the task, learning was rated less boring than the nonlearning situation $(2.7 \pm 0.3 \mathrm{vs}$ $4.0 \pm 0.4 ; p<.01)$. On self-rated sleepiness $(2.3 \pm 0.3$ vs $2.4 \pm$ $0.5)$, tiredness $(2.6 \pm 0.4$ vs $2.4 \pm 0.4)$, and tenseness $(2.1 \pm 0.2$ vs $2.0 \pm 0.1)$ after performing the 1 -hr-long task, again, both tasks did not show any differential effects $(p>0.50)$.

Density of sleep spindles was determined during sleep after learning and nonlearning tasks separately for each sleep stage and each of the five $90 \mathrm{~min}$ periods of sleep time. Mean spindle density was highest at the $\mathrm{Cz}$ electrode position over both conditions and all 90 min periods, with $4.2 \pm 0.7$ spindles per epoch (epoch length: $30 \mathrm{sec}$ ) during S2, $3.3 \pm 0.8$ during SWS, and $0.2 \pm$ 0.2 during REM sleep (Fz: $3.3 \pm 0.7,3.2 \pm 1.0$, and $0.2 \pm 0.003$, respectively). Results for other electrodes were similar, but spindle density decreased with distance from vertex; $<0.2$ spindles per epoch were detected on temporal electrodes (T3, T4). During S2, spindle density was significantly higher after the learning condition than after the nonlearning condition. The difference was largest at $\mathrm{Fz}$ and during the first 90 min after sleep onset $(3.9 \pm$ 0.8 vs $3.1 \pm 0.7, p<0.01 ; \mathrm{Cz}: 4.6 \pm 0.7$ vs $4.0 \pm 0.6, p=0.06$ ) (Fig. $1)$. This increase in spindle density could be found consistently in most of the subjects (Fig. $2 A$ ). Throughout the night, this difference in S2 spindle density diminished and was nonsignificant during the last $90 \mathrm{~min}$ of sleep (Fz: $3.3 \pm 0.8$ vs $3.1 \pm 0.8 ; p>$ 0.55). During SWS only a small, nonsignificant increase and during REM sleep no increase in spindle density was found. A notable finding is that spindle density correlated significantly with recall performance, both before and after sleep (Cz: $r=0.56$ before, $r=0.57$ after, both $p<0.05$; Fz: $r=0.52$ before, $r=0.53$ after, both $p=0.06)$.

Power spectral analysis revealed no differences between experimental conditions (Fig. 2B). Main effects for "learning versus nonlearning" and two- and three-way interactions between condition (learning vs nonlearning) and the factors "frequency band" ( $\delta: 1-4 \mathrm{~Hz} ; \theta$ : 4-8 Hz; $\alpha$ : 8-12 Hz; $\sigma: 12-15 \mathrm{~Hz} ; \beta$ : $15-20 \mathrm{~Hz}$ ) and "sleep stage" (S2 sleep, SWS, REM sleep) remained nonsignificant over all 28 EEG channels (all $p>0.10)$. This was confirmed by $t$ tests between both conditions over all channels and frequency 


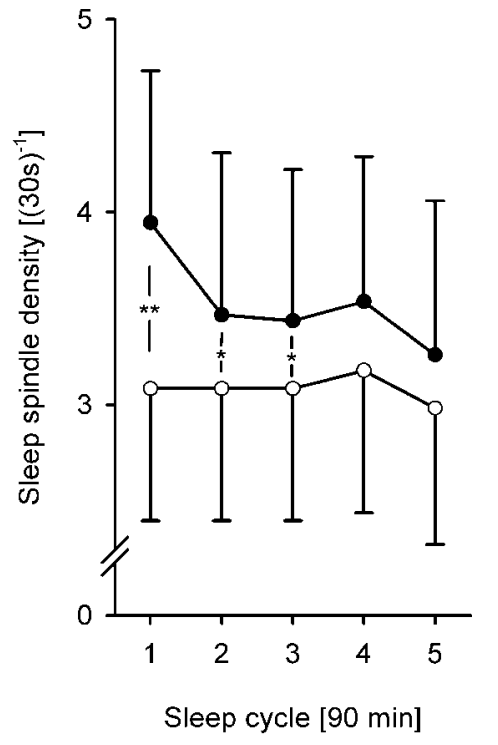

Figure 1. Mean number of sleep spindles at Fz per $30 \mathrm{sec}$ epoch during S2 in each 90 min sleep period over the course of the night. Filled circles represent the learning, and open circles represent the nonlearning condition. There is a notable decrease in spindle density over the night after learning $(p<0.01)$, which cannot be observed in the nonlearning condition $(p>0.50)$. Also, learning and nonlearning conditions differ significantly during the first three 90 min periods $\left({ }^{* *} p<0.01 ;{ }^{*} p<0.05\right)$. Large SEM values are attributable to the individual differences in spindle density, not to differences between conditions.

bands, which also remained nonsignificant (except for a higher delta band power during S2 sleep after learning at Oz, $p<0.05$ ).

\section{DISCUSSION}

After performing a declarative learning task for $1 \mathrm{hr}$, human subjects show an increased density of sleep spindles during S2 sleep in the following night, as compared with a nonlearning control task of comparable cognitive strain. This finding indicates that the learning experience before sleep can selectively modulate brain activity involved in subsequent spindle generation. There are only a few other studies that show experience-dependent changes in sleep EEG in humans. One study by Kattler et al. (1994) showed an increase in delta frequency activity over the somatosensory cortex after prolonged stimulation of the right hand. Our findings additionally confirm the hypothesis based on previous studies (Smith and MacNeill, 1994; Meier-Koll et al., 1999), that declarative memory function is related to S2 sleep activity, especially sleep spindles. The mean increase in spindle activity of $33.5 \%$ stands out against a considerable interindividual variance in spindle density that appears to be partly related to differences in learning capabilities. Suggesting such an association is the correlation between spindle density and recall performance, which indicates that better learners have higher spindle densities. Also, other influences, such as stimuli during the day before the beginning of the experiment, may have contributed to the individual variability by increasing spindle density in some of the nonlearning conditions.

Sleep spindle generation and declarative memory are located in different brain systems. Sleep spindles are generated in corticothalamic circuitries and originate from the nucleus reticularis of the thalamus (Steriade et al., 1993b). Declarative memory initially requires the hippocampus, and afterwards its contents are transferred to the neocortex for long-term storage (Buzsaki, 1998).
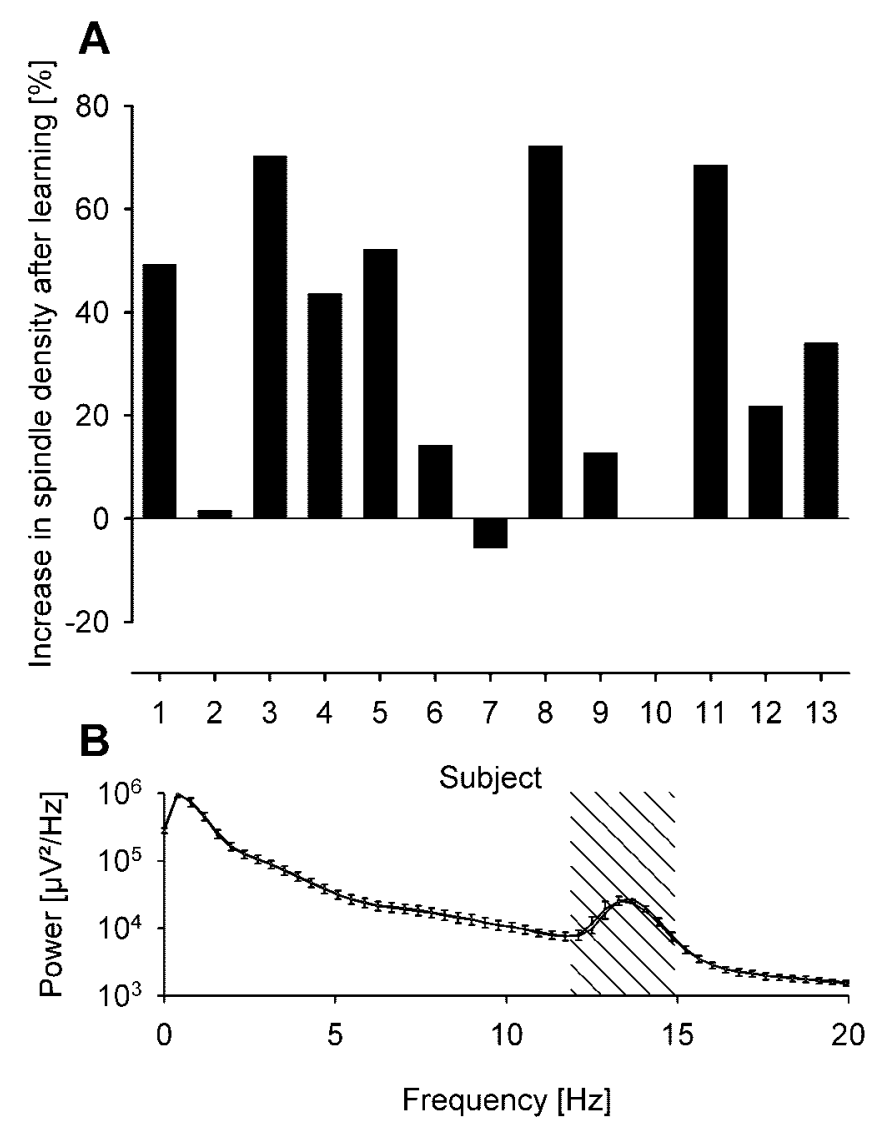

Figure 2. A, Individual increases (in percent) in spindle density at $\mathrm{Fz}$ during S2 of the first 90 min of sleep after learning as compared with the nonlearning condition. Most subjects show a substantially higher spindle density after learning. Mean increase $( \pm$ SEM) was $33.5 \pm 7.8 \%$, and a decrease of $\sim 5 \%$ can only be observed in one subject. $B$, Power spectra of all night $\mathrm{S} 2$ sleep at $\mathrm{Fz}$ in learning (error bars upward) and nonlearning (error bars downward) conditions. The hatched area indicates the spindle frequency band $(12-15 \mathrm{~Hz})$. Both curves are nearly identical, and differences are not significant. Higher spindle density during sleep after learning does not relate to greater power in the spindle frequency band.

Yet, thalamocortically generated sleep spindles (or their experimental model, i.e., augmenting thalamocortical responses) seem to be associated with these memory processes in the neocortex and hippocampus. Spindles have been shown to increase neocortical plasticity and neocortical synaptic responses, thus promoting memory consolidation (Steriade, 2001a,b). Furthermore, by means of local field potentials and single cell recordings, Siapas and Wilson (1998) showed in rats that hippocampal ripple activity tends to occur in temporal correlation to neocortical sleep spindles. Both systems therefore appear to be linked, presumably via some common modulating influence from another brain region coordinating the timing of these phenomena. (Note, however, that spindles can appear also in the absence of such modulations, even in the isolated thalamus.) Other studies could relate hippocampal sharp wave-ripple events to memory reprocessing (Chrobak et al., 2000; Draguhn et al., 2000). From studies in rats, it is known that during sleep those hippocampal neurons are reactivated that were active during a previous learning task (Wilson and McNaughton, 1994). This reactivation was mostly observed during non-REM sleep (Sutherland and McNaughton, 2000), although also REM sleep-associated reprocessing has been observed in one study (Louie and Wilson, 2001). It has to be 
noted here, that the studies cited above have been done in animal subjects and that similar processes in humans have yet to be demonstrated. Note also, that in the abovementioned animal studies, non-REM stages SWS and S2 are not differentiated, because in rodents these sleep stages are not as readily separable as they are in human subjects. In fact, the occurrence of spindles in rodents is commonly regarded as a sign of SWS. Together, the mechanisms suggested by the animal data provide a viable, although not yet experimentally proven, hypothesis for explaining the relationship between declarative memory reprocessing and cortical spindle events.

It might be noticed that the data show the largest increase in spindle density after learning at the Fz electrode, although the highest density of spindles was found at the $\mathrm{Cz}$ electrode. This finding can be interpreted in line with the findings of Werth et al. (1997) and Anderer et al. (2001), who could differentiate a frontal and a parietal source of sleep spindles. Thus, spindles recorded at $\mathrm{Cz}$ have the highest density because they represent the sum of frontal and parietal spindle activity. Mainly frontal spindles seem to be augmented after learning, and therefore the highest difference after learning can be observed at that lead.

Increased spindle activity might reflect self-sustained cortical activity after learning, eventually priming entorhinal and hippocampal circuitries. As an alternative interpretation, it has been proposed that based on previous learning experience, hippocampal activity selectively primes certain cortical circuits, which then participate in sleep spindle activity (Siapas and Wilson, 1998). The spindle activation itself may then provide the conditions necessary to induce long-term synaptic plastic changes, such as the emergence of a strong dipole within neocortical pyramidal cells (i.e., depolarization of apical dendrites in conjunction with hyperpolarization around the soma), $\mathrm{Ca}^{2+}$ entry into cortical dendrites, and the activation of the calcium-calmodulin kinase II (Sejnowski and Destexhe, 2000; Lisman et al., 2002). Sleep spindles may therefore, in conjunction with hippocampal ripples, be one factor serving eventually the consolidation of memories.

The data we present here do not prove the causal relationship between spindles and memory consolidation because they are just correlational in nature. However, the tasks were designed to be equal regarding visual input and cognitive strain and to differ only regarding the presence or absence of a long-term memory component. The increased spindle density in the learning condition as compared with the nonlearning condition therefore cannot be attributed to the visual input or other stimulus related aspects of the tasks. As cognitive strain and wearisomeness also did not differ between conditions, it is improbable that the altered sleep spindle activity is simply a result of differential fatigue. Therefore, the data speak strongly for an involvement of some common brain mechanism in both declarative long-term memory and spindle generation. The correlation between memory performance and spindle density further supports this conclusion, although it is not clear, whether this correlation reflects efficacy of the learning process before sleep or of memory consolidation during sleep.

Interestingly, the increase in spindle density was not associated with a general increase in band power in the spindle frequency band (12-15 Hz) after learning (Fig. 2B). This means that overall $12-15 \mathrm{~Hz}$ activity was not changed by previous learning activity, suggesting that the level of activity in the circuitries generating this rhythm is comparable during both conditions. However, 12-15 Hz activity after learning would be grouped into discrete spindles, as compared with a spread of activity in time after the nonlearning condition. This post hoc interpretation clearly is in need of further experimental validation. Also, analysis of band power in all other frequency bands did not show any other difference between the learning and nonlearning conditions. One hour of intense learning before sleep is therefore not enough to produce detectable changes in the standard EEG frequency bands. This may indicate that sleep does not simply reactivate oscillatory patterns occurring in the same frequency bands during waking (e.g., a decreased $\alpha$ activity and concurrently an increased theta activity as found by Mölle et al., 2002 for intentional learning). Rather, based upon previous learning experience, sleep appears to provide special conditions (e.g., neuromodulator levels) and mechanisms such as spindle activity to process newly acquired memory traces.

\section{REFERENCES}

Anderer P, Klosch G, Gruber G, Trenker E, Pascual-Marqui RD, Zeitlhofer J, Barbanoj MJ, Rappelsberger P, Saletu B (2001) Lowresolution brain electromagnetic tomography revealed simultaneously active frontal and parietal sleep spindle sources in the human cortex. Neuroscience 103:581-592.

Buzsaki G (1998) Memory consolidation during sleep: a neurophysiological perspective. J Sleep Res 7:17-23.

Chrobak JJ, Buzsaki G (1996) High-frequency oscillations in the output networks of the hippocampal- entorhinal axis of the freely behaving rat. J Neurosci 16:3056-3066.

Chrobak JJ, Lorincz A, Buzsaki G (2000) Physiological patterns in the hippocampo-entorhinal cortex system. Hippocampus 10:457-465.

Draguhn A, Traub RD, Bibbig A, Schmitz D (2000) Ripple (approximately $200-\mathrm{Hz}$ ) oscillations in temporal structures. J Clin Neurophysiol 17:361-376.

Gais S, Plihal W, Wagner U, Born J (2000) Early sleep triggers memory for early visual discrimination skills. Nat Neurosci 3:1335-1339.

Giuditta A, Ambrosini MV, Montagnese P, Mandile P, Cotugno M, Grassi ZG, Vescia S (1995) The sequential hypothesis of the function of sleep. Behav Brain Res 69:157-166.

Gratton G, Coles MG, Donchin E (1983) A new method for off-line removal of ocular artifact. Electroencephalogr Clin Neurophysiol 55:468-484.

Hasselmo ME (1999) Neuromodulation: acetylcholine and memory consolidation. Trends Cogn Sci 3:351-359.

Kattler H, Dijk DJ, Borbely AA (1994) Effect of unilateral somatosensory stimulation prior to sleep on the sleep EEG in humans. J Sleep Res 3:159-164.

Kudrimoti HS, Barnes CA, McNaughton BL (1999) Reactivation of hippocampal cell assemblies: effects of behavioral state, experience, and EEG dynamics. J Neurosci 19:4090-4101.

Lisman J, Schulman H, Cline H (2002) The molecular basis of CaMKII function in synaptic and behavioural memory. Nat Rev Neurosci 3:179-190.

Louie K, Wilson MA (2001) Temporally structured replay of awake hippocampal ensemble activity during rapid eye movement sleep. Neuron 29:145-156.

Meier-Koll A, Bussmann B, Schmidt C, Neuschwander D (1999) Walking through a maze alters the architecture of sleep. Percept Mot Skills 88:1141-1159.

Mölle M, Marshall L, Fehm HL, Born J (2002) EEG theta synchronization conjoined with alpha desynchronization indicate intentional encoding. Eur J Neurosci 15:923-928.

Nadasdy Z, Hirase H, Czurko A, Csicsvari J, Buzsaki G (1999) Replay and time compression of recurring spike sequences in the hippocampus. J Neurosci 19:9497-9507.

Plihal W, Born J (1997) Effects of early and late nocturnal sleep on declarative and procedural memory. J Cogn Neurosci 9:534-547.

Plihal W, Born J (1999) Effects of early and late nocturnal sleep on priming and spatial memory. Psychophysiology 36:571-582.

Qin YL, McNaughton BL, Skaggs WE, Barnes CA (1997) Memory reprocessing in corticocortical and hippocampocortical neuronal ensembles. Philos Trans R Soc Lond B Biol Sci 352:1525-1533.

Rechtschaffen A, Kales A (1968) A manual of standardized terminology, techniques and scoring system for sleep stages of human subjects. Los Angeles: Brain Information Service, University of California.

Sejnowski TJ, Destexhe A (2000) Why do we sleep? Brain Res 886:208-223.

Siapas AG, Wilson MA (1998) Coordinated interactions between hippocampal ripples and cortical spindles during slow-wave sleep. Neuron 21:1123-1128. 
Smith C, MacNeill C (1994) Impaired motor memory for a pursuit rotor task following Stage 2 sleep loss in college students. J Sleep Res 3:206-213.

Steriade M (2001a) Impact of network activities on neuronal properties in corticothalamic systems. J Neurophysiol 86:1-39.

Steriade M (2001b) The intact and sliced brain. Cambridge, MA: MIT.

Steriade M, Contreras D, Curro DR, Nunez A (1993a) The slow $(<1$ $\mathrm{Hz}$ ) oscillation in reticular thalamic and thalamocortical neurons: scenario of sleep rhythm generation in interacting thalamic and neocortical networks. J Neurosci 13:3284-3299.

Steriade M, McCormick DA, Sejnowski TJ (1993b) Thalamocortical oscillations in the sleeping and aroused brain. Science 262:679-685.
Sutherland GR, McNaughton B (2000) Memory trace reactivation in hippocampal and neocortical neuronal ensembles. Curr Opin Neurobiol 10:180-186.

Werth E, Achermann P, Dijk DJ, Borbely AA (1997) Spindle frequency activity in the sleep EEG: individual differences and topographic distribution. Electroencephalogr Clin Neurophysiol 103:535-542.

Wilson MA, McNaughton BL (1994) Reactivation of hippocampal ensemble memories during sleep. Science 265:676-679.

Ylinen A, Bragin A, Nadasdy Z, Jando G, Szabo I, Sik A, Buzsaki G (1995) Sharp wave-associated high-frequency oscillation $(200 \mathrm{~Hz})$ in the intact hippocampus: network and intracellular mechanisms. J Neurosci 15:30-46. 